\title{
Towards the Jacquet Conjecture on the Local Converse Problem for $p$-adic $\mathrm{GL}_{n}$
}

\author{
Dihua Jiang Chufeng Nien Shaun Stevens
}

\begin{abstract}
The Local Converse Problem is to determine how the family of the local gamma factors $\gamma(s, \pi \times \tau, \psi)$ characterizes the isomorphism class of an irreducible admissible generic representation $\pi$ of $\mathrm{GL}_{n}(F)$, with $F$ a non-archimedean local field, where $\tau$ runs through all irreducible supercuspidal representations of $\mathrm{GL}_{r}(F)$ and $r$ runs through positive integers. The Jacquet conjecture asserts that it is enough to take $r=1,2, \ldots,\left[\frac{n}{2}\right]$. Based on arguments in the work of Henniart and of Chen giving preliminary steps towards the Jacquet conjecture, we formulate a general approach to prove the Jacquet conjecture. With this approach, the Jacquet conjecture is proved under an assumption which is then verified in several cases, including the case of level zero representations.
\end{abstract}

Keywords. Irreducible admissible representation, Whittaker model, Local gamma factor, Local converse theorem

\section{Introduction}

Let $\pi$ be an irreducible (admissible) generic representation of $\mathrm{G}_{n}:=\mathrm{GL}_{n}(F)$, where $F$ is a locally compact non-archimedean local field. We may assume that $n \geqslant 2$, since the discussion in this paper for $n=1$ is trivial. Attached to $\pi$ is the family of local gamma factors $\gamma(s, \pi \times \tau, \psi)$, with $\tau$ any irreducible generic admissible representations of any $\mathrm{G}_{r}$, in the sense of Jacquet, Piatetski-Shapiro and Shalika ([13]), which can also be defined through the Langlands-Shahidi method ([19]). Here $\psi$ is a nontrivial additive character

D. Jiang: School of Mathematics, University of Minnesota, Minneapolis, MN 55455, USA; e-mail: dhjiang@math.umn.edu

C. Nien: Department of Mathematics, National Cheng Kung University and National Center for Theoretical Sciences (South), Tainan 701, Taiwan; e-mail: nienpig@ mail.ncku.edu.tw

S. Stevens: School of Mathematics, University of East Anglia, Norwich Research Park, Norwich, NR4 7TJ, UK; e-mail: Shaun.Stevens@uea.ac.uk

Mathematics Subject Classification (2010): Primary 11F70, 22E50; Secondary 11F85, 22E55 
of $F$; the definition of this family of local gamma factors is recalled in Section 2. It is natural to ask how this family of invariants yields information about the representation $\pi$.

In this paper, we consider the Local Converse Problem for $\mathrm{G}_{n}$, which is to find the least integer $n_{0}$ such that the family of local gamma factors $\gamma(s, \pi \times \tau, \psi)$, with $\tau$ running through all irreducible generic representations of $\mathrm{G}_{r}$ for $r=1, \ldots, n_{0}$, determines the irreducible generic representation $\pi$ of $\mathrm{G}_{n}$ up to isomorphism. It is an easy consequence of the work of Jacquet, Piatetski-Shapiro and Shalika ([13]) that $n_{0} \leqslant n$. The work of Henniart ([11]) shows that $n_{0} \leqslant n-1$, and the works of J.-P. Chen ([7] and [8]) and Cogdell and Piatetski-Shapiro ([9]) show that $n_{0} \leqslant n-2$, for $n \geqslant 3$. A stronger statement (when $n>4$ ) is the following conjecture, which is usually attributed to H. Jacquet.

Conjecture 1.1 (The Jacquet Conjecture on the Local Converse Problem). Let $\pi_{1}$ and $\pi_{2}$ be irreducible generic smooth representations of $\mathrm{G}_{n}$. If their local gamma factors $\gamma\left(s, \pi_{1} \times\right.$ $\tau, \psi)$ and $\gamma\left(s, \pi_{2} \times \tau, \psi\right)$ are equal, as functions in the complex variable $s$, for all irreducible generic representations $\tau$ of $\mathrm{G}_{r}$, with $r=1, \ldots,\left[\frac{n}{2}\right]$, then $\pi_{1}$ and $\pi_{2}$ are equivalent as representations of $\mathrm{G}_{n}$.

It is clear that the work $([11,7,9,8])$ confirms that for $2 \leqslant n \leqslant 4$, Conjecture 1.1 is a theorem. Indeed, for $n=2$, Conjecture 1.1 was proved in 1970 by Jacquet and Langlands in their well-known book ([12]), and for $n=3$ it was proved in 1979 by Jacquet, PiatetskiShapiro and Shalika ([14]). Following a standard argument, which was already known to the experts in the 1980s, we deduce in Section 2.4 that Conjecture 1.1 is equivalent to the following conjecture.

Conjecture 1.2. Assume that $\pi_{1}$ and $\pi_{2}$ are irreducible unitarizable supercuspidal representations of $\mathrm{G}_{n}$. If their local gamma factors $\gamma\left(s, \pi_{1} \times \tau, \psi\right)$ and $\gamma\left(s, \pi_{2} \times \tau, \psi\right)$ are equal as functions in the complex variable s, for all irreducible supercuspidal representations $\tau$ of $\mathrm{G}_{r}$ with $r=1, \ldots,\left[\frac{n}{2}\right]$, then $\pi_{1}$ and $\pi_{2}$ are equivalent as representations of $\mathrm{G}_{n}$.

Since any irreducible supercuspidal representation of $G_{n}$ has a nontrivial Whittaker model, it is natural to use this property, combined with the local functional equation of the local Rankin-Selberg convolution for $\mathrm{G}_{n} \times \mathrm{G}_{r}$, to figure out a possible approach to prove Conjecture 1.2. This is in fact the idea behind the previous attacks on the Local Converse Problem $([14,11,7,8])$. In this paper, we add a new idea to the argument in order to attempt to reduce the twists down to $r=1, \ldots,\left[\frac{n}{2}\right]$, i.e. Conjecture 1.2. The idea is to find Whittaker functions satisfying some special properties.

Let $\mathrm{U}_{n}$ be the unipotent radical of the standard Borel subgroup $\mathrm{B}_{n}$ of $\mathrm{G}_{n}$, which consists of all upper-triangular matrices. Denote by $\mathrm{P}_{n}$ the mirabolic subgroup of $\mathrm{G}_{n}$, consisting of matrices with last row equal to $(0, \ldots, 0,1)$. We also fix a standard non-degenerate character $\psi_{n}$ of $\mathrm{U}_{n}$ (see Section 2.1) so that all Whittaker functions are implicitly $\psi_{n^{-}}$ Whittaker functions. 
Definition 1.3. Let $\pi$ be an irreducible unitarizable supercuspidal representations of $\mathrm{G}_{n}$ and let $\mathrm{K}$ be a compact-mod-centre open subgroup of $\mathrm{G}_{n}$. A (non-zero) Whittaker function $W_{\pi}$ for $\pi$ is called $\mathbf{K}$-special if it satisfies:

$$
W_{\pi_{i}}\left(g^{-1}\right)=\overline{W_{\pi_{i}}(g)} \text { for all } g \in \mathbf{K},
$$

and $\operatorname{Supp} W_{\pi} \subset \mathrm{U}_{n} \mathbf{K}$, where ${ }^{-}$denotes complex conjugation.

Definition 1.4. Let $\pi_{1}$ and $\pi_{2}$ be irreducible unitarizable supercuspidal representations of $\mathrm{G}_{n}$ with the same central character. Let $W_{\pi_{1}}$ and $W_{\pi_{2}}$ be (non-zero) Whittaker functions for $\pi_{1}$ and $\pi_{2}$, respectively. We call $\left(W_{\pi_{1}}, W_{\pi_{2}}\right)$ a special pair (of Whittaker functions) for $\left(\pi_{1}, \pi_{2}\right)$ if there exists a compact-mod-centre open subgroup $\mathbf{K}$ of $\mathbf{G}_{n}$ such that $W_{\pi_{1}}$ and $W_{\pi_{2}}$ are both $\mathbf{K}$-special and

$$
W_{\pi_{1}}(p)=W_{\pi_{2}}(p), \text { for all } p \in \mathrm{P}_{n} .
$$

If a special pair of Whittaker functions as in Definition 1.4 exists for $\left(\pi_{1}, \pi_{2}\right)$, we can prove that the representations $\pi_{1}$ and $\pi_{2}$ are distinguished by their families of local gamma factors $\gamma\left(s, \pi_{i} \times \tau, \psi\right)$, for $\tau$ irreducible supercuspidal representations of $\mathrm{G}_{r}$, with $r=1, \ldots,\left[\frac{n}{2}\right]$, by using a refinement of the argument in [7] and [8]. This approach was successfully carried out by the second-named author in [17] for general linear groups over finite fields. The key point is to find a refined decomposition for $\mathrm{G}_{n}$ which reflects the symmetry carried in Definition 1.3. We recall in Section 3.1 this refined decomposition.

Theorem 1.5. Let $\pi_{1}$ and $\pi_{2}$ be irreducible unitarizable supercuspidal representations of $\mathrm{G}_{n}$. Assume that a special pair $\left(W_{\pi_{1}}, W_{\pi_{2}}\right)$ exists for $\left(\pi_{1}, \pi_{2}\right)$. If the local gamma factors $\gamma\left(s, \pi_{1} \times \tau, \psi\right)$ and $\gamma\left(s, \pi_{2} \times \tau, \psi\right)$ are equal as functions in the complex variable $s$, for all irreducible supercuspidal representations $\tau$ of $\mathrm{G}_{r}$ with $r=1, \ldots,\left[\frac{n}{2}\right]$, then $W_{\pi_{1}}=$ $W_{\pi_{2}}$ and $\pi_{1}$ and $\pi_{2}$ are equivalent as representations of $\mathrm{G}_{n}$.

In certain cases, one can prove the existence of special pairs for irreducible unitarizable supercuspidal representations of $\mathrm{G}_{n}$ by using the construction of supercuspidal representations in terms of maximal simple types of Bushnell and Kutzko ([6]) and the explicit construction of Bessel functions of supercuspidal representations due to Paškūnas and the third-named author ([18]). Given an irreducible supercuspidal representation $\pi$ of $\mathrm{G}_{n}$, one of the invariants associated to it, by Bushnell and Henniart in [2], is its endo-class $\Theta(\pi)$. We prove:

Proposition 1.6. Let $\pi_{1}, \pi_{2}$ be irreducible unitarizable supercuspidal representations of $\mathrm{G}_{n}$ with the same endo-class. Then there is a special pair $\left(W_{\pi_{1}}, W_{\pi_{2}}\right)$ for $\left(\pi_{1}, \pi_{2}\right)$.

Theorem 1.5 with Proposition 1.6 implies, for example, that two level zero irreducible unitarizable supercuspidal representations $\pi_{1}, \pi_{2}$ of $\mathrm{G}_{n}$ can be distinguished by the set of 
local gamma factors $\gamma\left(s, \pi_{i} \times \tau, \psi\right)$, for all irreducible supercuspidal representations $\tau$ of $\mathrm{G}_{r}$ with $r=1,2, \ldots,\left[\frac{n}{2}\right]$. In fact, this is a special case of a more general result, as follows.

Attached to an irreducible supercuspidal representation $\pi$ of $G_{n}$, via its endo-class $\Theta(\pi)$, is an invariant which we call its degree $\operatorname{deg}(\pi)$. The degree is an integer dividing $n$ : for example, $\operatorname{deg}(\pi)=1$ if and only if $\pi$ is a twist of a level zero representation; and if $\operatorname{deg}(\pi)<n$ then $\pi$ is invariant under a non-trivial unramified character twist, though the converse is not true. By using formulae on the conductors of pairs of supercuspidal representations from $[5,4]$, we immediately obtain the following corollary.

Corollary 1.7. Let $\pi_{1}$ and $\pi_{2}$ be irreducible unitarizable supercuspidal representations of $\mathrm{G}_{n}$ and suppose that $\operatorname{deg}\left(\pi_{1}\right)<n$. If the local gamma factors $\gamma\left(s, \pi_{1} \times \tau, \psi\right)$ and $\gamma\left(s, \pi_{2} \times\right.$ $\tau, \psi)$ are equal as functions in the complex variable s, for all irreducible supercuspidal representations $\tau$ of $\mathrm{G}_{r}$ with $r=1, \ldots,\left[\frac{n}{2}\right]$, then $\pi_{1}$ and $\pi_{2}$ are equivalent as representations of $\mathrm{G}_{n}$.

We end the paper with some discussion of the scope of the methods used here, in particular of the obstacles to extending to the case $\operatorname{deg}\left(\pi_{1}\right)=n$ (see Remark 5.3).

\section{Basics in the local Rankin-Selberg convolution}

We start by recalling the basic facts about Whittaker models of irreducible generic representations of $\mathrm{G}_{n}$ and local gamma factors of Rankin-Selberg convolution type over the locally compact non-archimedean local field $F$. We denote by $\mathfrak{o}_{F}$ the ring of integers in $F$, by $\mathfrak{p}_{F}$ the prime ideal in $\mathfrak{o}_{F}$, and by $\mathfrak{k}_{F}$ the residue field of $F$, of cardinality $q$ and characteristic $p$; we also write $|\cdot|$ for the absolute value on $F$, normalized to have image $q^{\mathbb{Z}}$. We use analogous notation for extensions of $F$. We also fix, once and for all, an additive character $\psi$ of $F$ which is trivial on $\mathfrak{p}_{F}$ but nontrivial on $\mathfrak{o}_{F}$.

\subsection{Whittaker models}

Let $\mathrm{Q}_{n}$ be the standard parabolic subgroup of $\mathrm{G}_{n}$ corresponding to the partition $(n-1,1)$. Then

$$
\mathrm{Q}_{n}=\mathrm{Z}_{n} \mathrm{P}_{n}
$$

where $\mathrm{Z}_{n}$ is the center of $\mathrm{G}_{n}$, and $\mathrm{P}_{n}$ is the mirabolic subgroup.

Definition 2.1. A character $\psi_{\mathrm{U}_{n}}$ of $\mathrm{U}_{n}$ is called non-degenerate if its normalizer in $\mathrm{B}_{n}$ is $\mathrm{Z}_{n} \mathrm{U}_{n}$. We denote by $\psi_{n}$ the standard non-degenerate character given by

$$
\psi_{n}(u)=\psi\left(\sum_{i=1}^{n-1} u_{i, i+1}\right)
$$


for $u=\left(u_{i, j}\right) \in \mathrm{U}_{n}$.

We call an irreducible smooth representation $\left(\pi, V_{\pi}\right)$ of $\mathrm{G}_{n}$ generic if there is a nondegenerate character $\psi_{\mathrm{U}_{n}}$ of $\mathrm{U}_{n}$ such that the Hom-space

$$
\operatorname{Hom}_{\mathrm{G}_{n}}\left(V_{\pi}, \operatorname{Ind}_{\mathrm{U}_{n}}^{\mathrm{G}_{n}}\left(\psi_{\mathrm{U}_{n}}\right)\right)
$$

is nonzero. By the uniqueness of local Whittaker models, this Hom-space is at most onedimensional. Since the non-degenerate characters of $\mathrm{U}_{n}$ are all conjugate under $\mathrm{B}_{n}$, we see that $\pi$ is generic if and only if

$$
\operatorname{Hom}_{\mathrm{G}_{n}}\left(V_{\pi}, \operatorname{Ind}_{\mathrm{U}_{n}}^{\mathrm{G}_{n}}\left(\psi_{n}\right)\right) \cong \operatorname{Hom}_{\mathrm{U}_{n}}\left(\left.V_{\pi}\right|_{\mathrm{U}_{n}}, \psi_{n}\right)
$$

is non-zero, where the isomorphism comes from Frobenius reciprocity.

Assume that $\pi$ is generic. We fix a nonzero functional $\ell$ in $\operatorname{Hom}_{\mathrm{U}_{n}}\left(\left.V_{\pi}\right|_{\mathrm{U}_{n}}, \psi_{n}\right.$ ) (which is unique up to scalar). The Whittaker function attached to a vector $v \in V_{\pi}$ is defined by

$$
W_{v}(g):=\ell(\pi(g)(v)), \text { for all } g \in \mathrm{G}_{n} .
$$

It is easy to see that $W_{v}$ belongs to $\operatorname{Ind}_{\mathrm{U}_{n}}^{\mathrm{G}_{n}}\left(\psi_{n}\right)$ and

$$
\mathcal{W}\left(\pi, \psi_{n}\right):=\left\{W_{v} \mid v \in V_{\pi}\right\}
$$

is called the $\psi_{n}$-Whittaker model of $\pi$, or simply the Whittaker model of $\pi$. It is clear that the Whittaker model of $\pi$ is independent of the choice of the nonzero functional $\ell$.

For any $W_{v} \in \mathcal{W}\left(\pi, \psi_{n}\right)$, define

$$
\widetilde{W}_{v}(g):=W_{v}\left(w_{n} \cdot{ }^{t} g^{-1}\right),
$$

for $g \in \mathrm{G}_{n}$, where $w_{n}$ is the longest Weyl group element of $\mathrm{G}_{n}$, with 1's on the second diagonal and zeros elsewhere, and ${ }^{t} g$ denotes the transpose of $g$. Then one can check that the function $\widetilde{W}_{v}$ belongs to the $\psi_{n}^{-1}$-Whittaker model of the contragredient $\widetilde{\pi}$ of $\pi$, that is,

$$
\widetilde{W}_{v} \in \mathcal{W}\left(\tilde{\pi}, \psi_{n}^{-1}\right) \subset \operatorname{Ind}_{\mathrm{U}_{n}}^{\mathrm{G}_{n}}\left(\psi_{n}^{-1}\right) .
$$

It is a basic fact that any irreducible supercuspidal representation of $G_{n}$ is generic ([10, Theorem B]). We recall the following properties of the restriction of an irreducible generic representation $\left(\pi, V_{\pi}\right)$ of $\mathrm{G}_{n}$ to the subgroup $\mathrm{P}_{n}$, which can be viewed as the starting point of our approach to prove the Jacquet conjecture for $\mathrm{G}_{n}$.

Theorem 2.2 ([1, §5]). With the notation fixed as above, the following hold.

(i) $\operatorname{Ind}_{\mathrm{U}_{n}}^{\mathrm{P}_{n}}\left(\psi_{n}\right)$ is irreducible as a representation of $\mathrm{P}_{n}$.

(ii) If $\pi$ is a generic representation of $\mathrm{G}_{n}$, then $\operatorname{Ind}_{\mathrm{U}_{n}}^{\mathrm{P}_{n}}\left(\psi_{n}\right)$ is a $\mathrm{P}_{n}$-subrepresentation of $\left.\mathcal{W}\left(\pi, \psi_{n}\right)\right|_{\mathrm{P}_{n}}$.

(iii) If $\pi$ is an irreducible supercuspidal representation of $\mathrm{G}_{n}$, then $\left.\pi\right|_{\mathrm{P}_{n}}$ is equivalent to $\operatorname{Ind}_{\mathrm{U}_{n}}^{\mathrm{P}_{n}}\left(\psi_{n}\right)$ as representations of $\mathrm{P}_{n}$. 


\subsection{Local gamma factors}

Next we review the basic setting of local gamma factors attached to a pair of irreducible generic representations, for details of which we refer to [13].

Let $n, r \geqslant 1$ be integers and let $\pi$ and $\tau$ be irreducible generic representations of $\mathrm{G}_{n}$ and $\mathrm{G}_{r}$, respectively, with central characters $\omega_{\pi}$ and $\omega_{\tau}$ respectively. Let $W_{\pi} \in \mathcal{W}\left(\pi, \psi_{n}\right)$ be a Whittaker function of $\pi$ and $W_{\tau} \in \mathcal{W}\left(\tau, \psi_{r}^{-1}\right)$ be a Whittaker function of $\tau$. Since it is the only case of interest to us here, we suppose that $n>r$.

If $j$ is an integer for which $n-r-1 \geqslant j \geqslant 0$, a local zeta integral for the pair $(\pi, \tau)$ is defined by

$$
\mathcal{Z}\left(W_{\pi}, W_{\tau}, s ; j\right):=\int_{g} \int_{x} W_{\pi}\left(\begin{array}{ccc}
g & 0 & 0 \\
x & \mathrm{I}_{j} & 0 \\
0 & 0 & \mathrm{I}_{n-r-j}
\end{array}\right) W_{\tau}(g)|\operatorname{det} g|^{s-\frac{n-r}{2}} d x d g
$$

where the integration in the variable $g$ is over $\mathrm{U}_{r} \backslash \mathrm{G}_{r}$ and the integration in the variable $x$ is over $\operatorname{Mat}_{j \times r}(F)$. Jacquet, Piatetski-Shapiro, and Shalika proved in [13] the following theorem.

For $g \in \mathrm{G}_{n}$, we denote by $R_{g}$ the right translation action of $g$ on functions from $\mathrm{G}_{n}$ to $\mathbb{C}$, and we put $w_{n, r}=\left(\begin{array}{cc}\mathrm{I}_{r} & 0 \\ 0 & w_{n-r}\end{array}\right)$.

Theorem 2.3 ([13, Section 2.7]). With notation as above, the following hold.

(i) Each integral $\mathcal{Z}\left(W_{\pi}, W_{\tau}, \Phi, s ; j\right)$ is absolutely convergent for $\operatorname{Re}(s)$ sufficiently large and is a rational function of $q^{-s}$. More precisely, for fixed $j$, the integrals $\mathcal{Z}\left(W_{\pi}, W_{\tau}, s ; j\right)$ span a fractional ideal (independent of $j$ )

$$
\mathbb{C}\left[q^{s}, q^{-s}\right] L(s, \pi \times \tau)
$$

of the ring $\mathbb{C}\left[q^{s}, q^{-s}\right]$, where the local $L$-factor $L(s, \pi \times \tau)$ has the form $P\left(q^{s}\right)^{-1}$, with $P \in \mathbb{C}[x]$ and $P(0)=1$.

(ii) For $n-r-1 \geqslant j \geqslant 0$, there is a factor $\epsilon(s, \pi \times \tau, \psi)$ independent of $j$, such that

$$
\begin{aligned}
\frac{\mathcal{Z}\left(R_{w_{n, r}} \widetilde{W}_{\pi}, \widetilde{W}_{\tau}, 1-s ; n-r-j-1\right)}{L(1-s, \widetilde{\pi} \times \widetilde{\tau})} \\
=\omega_{\tau}(-1)^{n-1} \epsilon(s, \pi \times \tau, \psi) \frac{\mathcal{Z}\left(W_{\pi}, W_{\tau}, s ; j\right)}{L(s, \pi \times \tau)} .
\end{aligned}
$$

(iii) There are $c \in \mathbb{C}^{\times}$and $f=f(\pi \times \tau, \psi) \in \mathbb{Z}$ such that

$$
\epsilon(s, \pi \times \tau, \psi)=c q^{-f s} .
$$


The local gamma factor attached to a pair of representations $\pi$ and $\tau$ is defined in [13] by

$$
\gamma(s, \pi \times \tau, \psi)=\epsilon(s, \pi \times \tau, \psi) \frac{L(1-s, \widetilde{\pi} \times \widetilde{\tau})}{L(s, \pi \times \tau)} .
$$

Then the functional equation in Part (ii) of Theorem 2.3 can be rewritten

$$
\mathcal{Z}\left(R_{w_{n, r}} \widetilde{W}_{\pi}, \widetilde{W}_{\tau}, 1-s ; n-r-j-1\right)=\omega_{\tau}(-1)^{n-1} \gamma(s, \pi \times \tau, \psi) \mathcal{Z}\left(W_{\pi}, W_{\tau}, s, j\right)
$$

We also remark that the local gamma factor $\gamma(s, \pi \times \tau, \psi)$ determines the conductor $f(\pi \times$ $\tau, \psi)$, since it is the leading power of $q^{-s}$ in a power series expansion for $\gamma(s, \pi \times \tau, \psi)$.

\subsection{Central characters}

In this section, we show that the well-known result that local gamma factors determine the central character. We begin by recalling the following result on the stability of local gamma factors, which follows from [15, Proposition 2.7].

Proposition 2.6. Let $\pi$ be an irreducible generic representation of $\mathrm{G}_{n}$ with $n \geq 2$. Then there exits $m_{\pi}$ such that, for any character $\chi$ of $F^{\times}$of conductor $m \geqslant m_{\pi}$ and any $c \in$ $\mathfrak{p}^{-m}$ satisfying $\chi(1+x)=\psi(c x)$, for $x \in \mathfrak{p}_{F}^{\left[\frac{m}{2}\right]+1}$, we have

$$
L(s, \pi \times \chi)=1 \text { and } \epsilon(s, \pi \times \chi, \psi)=\omega_{\pi}(c)^{-1} \epsilon(s, 1 \times \chi, \psi)^{n} .
$$

Proof. Although this is not quite the statement of [15, Proposition 2.7], this statement is included in the proof (see page 323 of op. cit.).

Corollary 2.7. Let $\pi_{1}, \pi_{2}$ be irreducible generic representations of $\mathrm{G}_{n}$. If their local gamma factors $\gamma\left(s, \pi_{1} \times \chi, \psi\right)$ and $\gamma\left(s, \pi_{2} \times \chi, \psi\right)$ are equal as functions in the complex variable $s$, for any character $\chi$ of $F^{\times}$, then $\omega_{\pi_{1}}=\omega_{\pi_{2}}$.

Proof. For $i=1,2$, let $m_{\pi_{i}}, m_{\widetilde{\pi}_{i}}$ be the numbers given by Proposition 2.6 and put $m_{0}=$ $\max \left\{m_{\pi_{i}}, m_{\widetilde{\pi}_{i}} \mid i=1,2\right\}$. For $\chi$ a character of $F^{\times}$of conductor $m \geqslant m_{0}$, we have $\epsilon\left(s, \pi_{i} \times\right.$ $\chi, \psi)=\gamma\left(s, \pi_{i} \times \chi, \psi\right)$, by (2.4) and Proposition 2.6.

For any $c \in \mathfrak{p}^{-m} \backslash \mathfrak{p}^{1-m}$, with $m \geqslant m_{0}$, there exists a character $\chi_{c}$ character of conductor $m$ such that $\chi_{c}(1+x)=\psi(c x)$, for $x \in \mathfrak{p}_{F}^{\left[\frac{m}{2}\right]+1}$; thus Proposition 2.6 implies

$$
\omega_{\pi_{1}}(c)=\omega_{\pi_{2}}(c)
$$

Since any element of $F^{\times}$can be expressed as the quotient of two elements of valuation at most $-m$, we deduce that $\omega_{\pi_{1}}=\omega_{\pi_{2}}$. 


\subsection{Reduction from generic to supercuspidal}

This section is devoted to reducing Conjecture 1.1 to Conjecture 1.2. In other words, if the Local Converse Theorem for twisting by generic representations of rank up to $\left[\frac{n}{2}\right]$ holds for unitarizable supercuspidal representations, then it also holds for general generic smooth representations.

Let $\pi$ be an irreducible generic smooth representation of $\mathrm{G}_{n}$. From the classification of irreducible smooth representations of $\mathrm{G}_{n}$ [20, Theorem 9.7], $\pi$ is the unique irreducible generic subquotient of a standard parabolically induced representation

$$
\tau_{1}|\cdot|^{z_{1}} \times \cdots \times \tau_{t}|\cdot|^{z_{t}}
$$

where each $\tau_{i}$ is an irreducible unitarizable supercuspidal representation of $\mathrm{G}_{n_{i}}$, with $n=$ $\sum_{i=1}^{t} n_{i}$, and

$$
z_{1} \geqslant \cdots \geqslant z_{t}
$$

are real numbers. Moreover $\left(\tau_{1}, \ldots, \tau_{t}\right)$ and $\left(z_{1}, \ldots, z_{t}\right)$ are uniquely determined up to a permutation $\sigma$ such that $z_{\sigma(i)}=z_{i}$, and any such tuples give rise to an irreducible generic representation of $\mathrm{G}_{n}$ in this way. By the multiplicativity of the local gamma factors ([13, Theorem 3.1]), we have

$$
\gamma(s, \pi \times \tau, \psi)=\prod_{i=1}^{t} \gamma\left(s+z_{i}, \tau_{i} \times \tau, \psi\right),
$$

for all irreducible generic representations $\tau$ of $\mathrm{G}_{r}$. We also observe that there is at most one index $i$ such that $n_{i}>\left[\frac{n}{2}\right]$.

Proposition 2.9 ([16, Section 3.2]). With notation as above, assume that $\tau$ is irreducible, unitarizable and supercuspidal.

(i) If $\prod_{i=1}^{t} \gamma\left(s+z_{i}, \tau_{i} \times \tau, \psi\right)$ has a real pole (respectively, zero) at $s=s_{0}$, then $\tau \simeq \widetilde{\tau}_{i}$ and $s_{0}=1-z_{i}$ (respectively, $\left.s_{0}=-z_{i}\right)$, for some $i \in\{1, \ldots, t\}$.

(ii) For each $j=1, \ldots, t$, the product $\prod_{i=1}^{t} \gamma\left(s+z_{i}, \tau_{i} \times \tilde{\tau}_{j}, \psi\right)$ has a real pole and zero. Moreover, if $j=1$ then there is a zero at $s=-z_{1}$, and if $j=t$ then there is $a$ pole at $s=1-z_{t}$.

Note that the assumption in [16], that $F$ is of characteristic zero, is not used in the proof of this since it requires only the multiplicativity of local gamma factors.

Corollary 2.10. With notation as above, suppose also that $\tau_{i}^{\prime}$ are irreducible unitarizable supercuspidal representation of $\mathrm{G}_{n_{i}^{\prime}}$, for $1 \leqslant i \leqslant t^{\prime}$, with $n=\sum_{i=1}^{t^{\prime}} n_{i}^{\prime}$, and that $z_{1}^{\prime} \geqslant$ $\cdots \geqslant z_{t^{\prime}}^{\prime}$ are real numbers. Suppose $m \geqslant\left[\frac{n}{2}\right]$ and

$$
\prod_{i=1}^{t} \gamma\left(s+z_{i}, \tau_{i} \times \tau, \psi\right)=\prod_{i=1}^{t^{\prime}} \gamma\left(s+z_{i}^{\prime}, \tau_{i}^{\prime} \times \tau, \psi\right),
$$


for all irreducible unitarizable supercuspidal representations $\tau$ of $\mathrm{G}_{r}$, with $r=1,2, \ldots, m$. Then $t=t^{\prime}$ and there is a permutation $\sigma$ of $\{1, \ldots t\}$ such that:

(i) $n_{i}=n_{\sigma(i)}^{\prime}$, for all $i=1, \ldots, t$;

(ii) $\gamma\left(s+z_{i}, \tau_{i} \times \tau, \psi\right)=\gamma\left(s+z_{\sigma(i)}^{\prime}, \tau_{\sigma(i)}^{\prime} \times \tau, \psi\right)$, for all irreducible unitarizable supercuspidal representations $\tau$ of $\mathrm{G}_{r}$, with $r=1,2, \ldots, m$ and $i=1, \ldots, t$;

(iii) $\tau_{i} \simeq \tau_{\sigma(i)}^{\prime}$ and $z_{i}=z_{\sigma(i)}^{\prime}$, for all $i$ such that $n_{i} \leqslant\left[\frac{n}{2}\right]$.

Proof. The proof is by induction on $t$. If $t=1$ but $t^{\prime}>1$ then $n_{j}^{\prime} \leqslant\left[\frac{n}{2}\right]$, for some $j$, and, by Proposition 2.9(i), $\prod_{i=1}^{t^{\prime}} \gamma\left(s+z_{i}^{\prime}, \tau_{i}^{\prime} \times \widetilde{\tau}_{j}^{\prime}, \psi\right)$ has a real pole while $\gamma\left(s+z_{1}, \tau_{1} \times \widetilde{\tau}_{j}^{\prime}, \psi\right)$ does not, which is absurd. Thus $t^{\prime}=1$ and there is nothing more to prove.

Now assume $t \geqslant 2$ and note that either $n_{1}$ or $n_{t}$ is at most $\left[\frac{n}{2}\right]$. Suppose first that $n_{t} \leqslant$ $\left[\frac{n}{2}\right]$. Then $\prod_{i=1}^{t} \gamma\left(s+z_{i}, \tau_{i} \times \widetilde{\tau}_{t}, \psi\right)$ has a pole at $1-z_{t}$ so, by Proposition 2.9(i), there is an integer $1 \leqslant j \leqslant t^{\prime}$ such that $\tau_{j}^{\prime} \simeq \tau_{t}$ and $z_{j}^{\prime}=z_{t}$. Hence $\tau_{j}^{\prime}|\cdot| z_{j}^{\prime} \simeq \tau_{t}|\cdot|{ }^{z_{t}}$ and $\gamma\left(s+z_{t}, \tau_{t} \times \tau, \psi\right)=\gamma\left(s+z_{j}^{\prime}, \tau_{j}^{\prime} \times \tau, \psi\right)$, for all irreducible generic representations $\tau$ of $G_{r}$, for all $r$. In particular, we deduce

$$
\prod_{i=1}^{t-1} \gamma\left(s+z_{i}, \tau_{i} \times \tau, \psi\right)=\prod_{i=1, i \neq j}^{t^{\prime}} \gamma\left(s+z_{i}^{\prime}, \tau_{i}^{\prime} \times \tau, \psi\right),
$$

for all irreducible unitarizable supercuspidal representations $\tau$ of $\mathrm{G}_{r}$, with $r=1,2, \ldots, m$. The result now follows from the inductive hypothesis.

Finally, if $n_{1} \leqslant\left[\frac{n}{2}\right]$ then $\prod_{i=1}^{t} \gamma\left(s+z_{i}, \tau_{i} \times \widetilde{\tau}_{1}, \psi\right)$ has a zero at $-z_{1}$ so, by Proposition 2.9(i), there is an integer $1 \leqslant j \leqslant t^{\prime}$ such that $\tau_{j}^{\prime} \simeq \tau_{1}$ and $z_{j}^{\prime}=z_{1}$. The result then follows as in the first case.

Putting Corollary 2.10 with $m=\left[\frac{n}{2}\right]$ together with the multiplicativity of local gamma factors (2.8) and the classification of irreducible generic representations [20, Theorem 9.7], we see that Conjecture 1.2 implies Conjecture 1.1.

\section{Special pairs and the local converse theorem}

\subsection{Preliminary results}

We begin by recalling some useful lemmas, which form the technical steps of the proof.

Lemma 3.1 ([15, Section 3.2]). Let t be a positive integer and let $H$ be a complex smooth function on $\mathrm{G}_{t}$ with compact support modulo $\mathrm{U}_{t}$ satisfying

$$
H(u g)=\psi_{t}(u) H(g)
$$


for all $u \in \mathrm{U}_{t}, g \in \mathrm{G}_{t}$. If

$$
\int_{\mathrm{U}_{t} \backslash \mathrm{G}_{t}} H(g) W_{\tau}(g) d g=0
$$

for all $W_{\tau} \in \mathcal{W}\left(\tau, \psi_{t}^{-1}\right)$, with $\tau$ running through all irreducible generic representations of $\mathrm{G}_{t}$, then $H \equiv 0$.

From [8, Section 3.1], we have the generalized Bruhat decomposition:

$$
\mathrm{G}_{n}=\bigsqcup_{i=0}^{n-1} \mathrm{U}_{n} \alpha^{i} \mathrm{Q}_{n}
$$

where $\alpha=\left(\begin{array}{cc}0 & \mathrm{I}_{n-1} \\ 1 & 0\end{array}\right)$.

Definition 3.2. Given two functions $H_{1}$ and $H_{2}$ on $\mathrm{G}_{n}$, if

$$
H_{1}(x)=H_{2}(x) \text {, for all } x \in \mathrm{U}_{n} \alpha^{i} \mathrm{Q}_{n},
$$

then we say that $H_{1}$ and $H_{2}$ agree on height $i$.

Assume that $\left(W_{\pi_{1}}, W_{\pi_{2}}\right)$ is a special pair for $\left(\pi_{1}, \pi_{2}\right)$, as in Definition 1.4, so that $W_{\pi_{1}}$ and $W_{\pi_{2}}$ agree on height $i=0$. The condition on local gamma factors in the statement Conjecture 1.2, via Corollary 2.7 and the following proposition, implies the agreement of $W_{\pi_{1}}$ and $W_{\pi_{2}}$ on height $i$, for $i=0, \ldots,\left[\frac{n}{2}\right]$.

Proposition 3.3 ([8, Proposition 3.1]). Fix an integer $1 \leqslant r<n$. Let $\pi_{1}$ and $\pi_{2}$ be irreducible supercuspidal representations of $\mathrm{G}_{n}$ with the same central character, and let $W_{\pi_{1}}, W_{\pi_{2}}$ be Whittaker functions, for $\pi_{1}, \pi_{2}$ respectively, which coincide on $\mathrm{P}_{n}$. If the local gamma factors $\gamma\left(s, \pi_{1} \times \tau, \psi\right)$ and $\gamma\left(s, \pi_{2} \times \tau, \psi\right)$ are equal as functions in the complex variable $s \in \mathbb{C}$, for all irreducible generic representations $\tau$ of $\mathrm{G}_{r}$, then the two Whittaker functions $W_{\pi_{1}}, W_{\pi_{2}}$ agree on height $r$.

We are going to use the functional equations together with the properties of special pairs of Whittaker functions in order to show that if a special pair $\left(W_{\pi_{1}}, W_{\pi_{2}}\right)$ agree on height $i$, for $i=0, \ldots,\left[\frac{n}{2}\right]$, then they are in fact equal. To do so, we apply a refined decomposition of $\mathrm{G}_{n}$, whose finite field version was a key ingredient in the proof of the Jacquet Conjecture on the Local Converse Problem for $G_{n}$ over finite fields in [17].

Proposition 3.4 ([17, Proposition 3.8]). The following (non-disjoint) decomposition holds:

$$
\mathrm{G}_{n}=\bigcup_{0 \leqslant r \leqslant\left[\frac{n}{2}\right], n-\left[\frac{n}{2}\right] \leqslant k \leqslant n} \mathrm{U}_{n} \alpha^{r} \mathrm{Q}_{n} \alpha^{k} \mathrm{U}_{n} .
$$




\subsection{Proof of Theorem 1.5}

Let $\pi_{1}, \pi_{2}$ be irreducible supercuspidal representations of $\mathrm{G}_{n}$ and let $\left(W_{\pi_{1}}, W_{\pi_{2}}\right)$ be a special pair for $\left(\pi_{1}, \pi_{2}\right)$. Let $\mathbf{K}$ be the compact-mod-centre open subgroup of $\mathrm{G}_{n}$ such that $W_{\pi_{i}}$ are K-special. By hypothesis, the local gamma factors $\gamma\left(s, \pi_{1} \times \tau, \psi\right)$ and $\gamma\left(s, \pi_{2} \times\right.$ $\tau, \psi)$ are equal as functions in the complex variable $s$, for all irreducible supercuspidal representations $\tau$ of $\mathrm{G}_{r}$ with $r=1, \ldots,\left[\frac{n}{2}\right]$. This condition can be extended for all irreducible generic smooth representations $\tau$ of $\mathrm{G}_{n}$ by the multiplicativity of local gamma factors. Moreover, by Corollary 2.7, $\pi_{1}, \pi_{2}$ have the same central character. The proof goes in three steps.

Step (1). By Proposition 3.3, $W_{\pi_{1}}(g)=W_{\pi_{2}}(g)$, for

$$
g \in \bigcup_{0 \leqslant r \leqslant\left[\frac{n}{2}\right]} \mathrm{U}_{n} \alpha^{r} \mathrm{Q}_{n}=\bigcup_{0 \leqslant r \leqslant\left[\frac{n}{2}\right]} \mathrm{U}_{n} \alpha^{r} \mathrm{Q}_{n} \alpha^{n} \mathrm{U}_{n}
$$

Step (2). For $g=q \alpha^{k} u \in \mathrm{Q}_{n} \alpha^{k} \mathrm{U}_{n} \cap \mathbf{K}$, with $n-\left[\frac{n}{2}\right] \leqslant k \leqslant n$, and $i=1,2$, we have

$$
W_{\pi_{i}}\left(q \alpha^{k} u\right)=\overline{W_{\pi_{i}}\left(\left(q \alpha^{k} u\right)^{-1}\right)}=\overline{W_{\pi_{i}}\left(u^{-1} \alpha^{n-k} q^{-1}\right)},
$$

since $W_{\pi_{i}}$ is $\mathbf{K}$-special. Since $u^{-1} \alpha^{n-k} q^{-1} \in \mathrm{U}_{n} \alpha^{n-k} \mathrm{Q}_{n}$, from Step (1) it follows that

$$
W_{\pi_{1}}\left(q \alpha^{k} u\right)=W_{\pi_{2}}\left(q \alpha^{k} u\right)
$$

Thus $W_{\pi_{1}}, W_{\pi_{2}}$ agree on $\mathrm{Q}_{n} \alpha^{k} \mathrm{U}_{n} \cap \mathbf{K}$ and hence on $\mathrm{Q}_{n} \alpha^{k} \mathrm{U}_{n} \cap \mathrm{U}_{n} \mathbf{K}$, since they are both $\psi_{n}$-Whittaker functions. Since $\operatorname{Supp} W_{\pi_{i}} \subset \mathrm{U}_{n} \mathbf{K}$, we deduce that $W_{\pi_{1}}(g)=W_{\pi_{2}}(g)$, for all

$$
g \in \bigcup_{n-\left[\frac{n}{2}\right] \leqslant k \leqslant n} \mathrm{Q}_{n} \alpha^{k} \mathrm{U}_{n}=\bigcup_{n-\left[\frac{n}{2}\right] \leqslant k \leqslant n} \mathrm{U}_{n} \alpha^{0} \mathrm{Q}_{n} \alpha^{k} \mathrm{U}_{n} .
$$

Step (3). It remains to consider the case of $g \in \mathrm{U}_{n} \alpha^{r} \mathrm{Q}_{n} \alpha^{k} \mathrm{U}_{n}$, with $1 \leqslant r \leqslant\left[\frac{n}{2}\right]$ and $n-\left[\frac{n}{2}\right] \leqslant k \leqslant n-1$. For any fixed $u \in \mathrm{U}_{n}$ and $p \in \mathrm{P}_{n}$, Step (2) implies that

$$
R_{p \alpha^{k} u} W_{\pi_{1}}(q)=R_{p \alpha^{k} u} W_{\pi_{2}}(q)
$$

for all $q \in \mathrm{P}_{n}$, where we recall that $R_{g}$ denotes the right translation action by $g$ on the Whittaker functions. We apply the functional equation (2.5) for $j=n-r-1$ to the Whittaker functions $R_{p \alpha^{k} u} W_{\pi_{i}}$ for $i=1,2$ and any Whittaker function $W_{\tau}$ in $\mathcal{W}\left(\tau, \psi_{r}^{-1}\right)$.

The local zeta function $\mathcal{Z}\left(R_{p \alpha^{k} u} W_{\pi_{i}}, W_{\tau}, s ; n-r-1\right)$ is given by the following integral

$$
\int_{h} \int_{x} R_{p \alpha^{k} u} W_{\pi_{i}}\left(\begin{array}{ccc}
h & 0 & 0 \\
x & \mathrm{I}_{n-r-1} & 0 \\
0 & 0 & 1
\end{array}\right) W_{\tau}(h)|\operatorname{det} h|^{s-\frac{n-r}{2}} d x d h
$$


where the integration in the variable $h$ is over $\mathrm{U}_{r} \backslash \mathrm{G}_{r}$ and the integration in the variable $x$ is over $\operatorname{Mat}_{(n-r-1) \times r}(F)$. Hence we obtain

$$
\mathcal{Z}\left(R_{p \alpha^{k} u} W_{\pi_{1}}, W_{\tau}, s ; n-r-1\right)=\mathcal{Z}\left(R_{p \alpha^{k} u} W_{\pi_{2}}, W_{\tau}, s ; n-r-1\right) .
$$

Since $\gamma\left(s, \pi_{1} \times \tau, \psi\right)=\gamma\left(s, \pi_{2} \times \tau, \psi\right)$, by the functional equation (2.5) for $j=n-r-1$, we obtain

$$
\mathcal{Z}\left(R_{w_{n, r}}{\widetilde{R_{p \alpha^{k} u} W_{\pi_{1}}}}_{\widetilde{W}_{\tau}}, 1-s ; 0\right)=\mathcal{Z}\left(R_{w_{n, r}}{\widetilde{R_{p \alpha^{k} u} W_{\pi_{2}}}}, \widetilde{W}_{\tau}, 1-s ; 0\right) .
$$

Thus, from the definition of these zeta integrals,

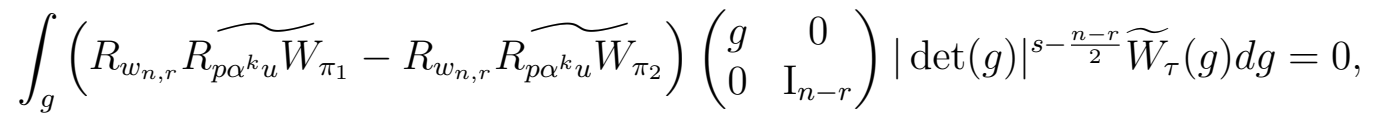

for all generic representations $\tau$ of $\mathrm{G}_{r}$, where the integration in the variable $h$ is over $\mathrm{U}_{r} \backslash \mathrm{G}_{r}$. From Lemma 3.1, we deduce that

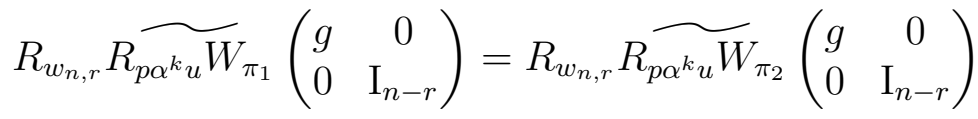

for all $p \in \mathrm{P}_{n}, u \in \mathrm{U}_{n}$ and $g \in \mathrm{G}_{r}$. Now by definition, for $i=1,2$,

$$
\begin{aligned}
R_{w_{n, r}} \widetilde{R_{p \alpha^{k} u} W_{\pi_{i}}}\left(\begin{array}{cc}
g & 0 \\
0 & \mathrm{I}_{n-r}
\end{array}\right) & =R_{p \alpha^{k} u} W_{\pi_{i}}\left(w_{n}\left(\begin{array}{cc}
{ }^{t} g^{-1} & 0 \\
0 & I_{n-r}
\end{array}\right){ }^{t} w_{n, r}^{-1}\right) \\
& =W_{\pi_{i}}\left(\left(\begin{array}{cc}
0 & \mathrm{I}_{n-r} \\
w_{r}{ }^{t} g^{-1} & 0
\end{array}\right) p \alpha^{k} u\right) .
\end{aligned}
$$

Hence we obtain the identity

$$
W_{\pi_{1}}\left(\left(\begin{array}{cc}
0 & \mathrm{I}_{n-r} \\
w_{r}^{t} g^{-1} & 0
\end{array}\right) p \alpha^{k} u\right)=W_{\pi_{2}}\left(\left(\begin{array}{cc}
0 & \mathrm{I}_{n-r} \\
w_{r}^{t} g^{-1} & 0
\end{array}\right) p \alpha^{k} u\right),
$$

for all $p \in \mathrm{P}_{n}, u \in \mathrm{U}_{n}$ and $g \in \mathrm{G}_{r}$. In particular, taking $g=w_{r}$ we obtain

$$
W_{\pi_{1}}\left(\alpha^{r} p \alpha^{k} u\right)=W_{\pi_{2}}\left(\alpha^{r} p \alpha^{k} u\right),
$$

for all $p \in \mathrm{P}_{n}$ and $u \in \mathrm{U}_{n}$. This proves that $W_{\pi_{1}}(g)=W_{\pi_{2}}(g)$, for

$$
g \in \mathrm{U}_{n} \alpha^{r} \mathrm{Q}_{n} \alpha^{k} \mathrm{U}_{n}
$$

with $1 \leqslant r \leqslant\left[\frac{n}{2}\right]$ and $n-\left[\frac{n}{2}\right] \leqslant k \leqslant n-1$. This completes Step (3).

By combining the results from all three Steps above, we obtain that

$$
W_{\pi_{1}}(g)=W_{\pi_{2}}(g) \text {, for all } g \in \mathrm{G}_{n} .
$$

By the uniqueness of local Whittaker models for irreducible smooth representations of $\mathrm{G}_{n}$, the two Whittaker models $\mathcal{W}\left(\pi_{1}, \psi_{n}\right)$ and $\mathcal{W}\left(\pi_{2}, \psi_{n}\right)$ have trivial intersection unless $\pi_{1}$ and $\pi_{2}$ are equivalent as representations of $\mathrm{G}_{n}$, which completes the proof of Theorem 1.5. 


\section{Supercuspidals with the same endo-class}

K-special Whittaker functions are Whittaker functions of $\mathrm{G}_{n}$ with certain symmetry when restricted to $K$. The Bessel functions of irreducible supercuspidal representations of $G_{n}$ constructed by Paškūnas and the third-named author in [18] are such examples. We recall from [18] the basics of these Bessel functions, which rely on the construction theory of supercuspidal representations of $\mathrm{G}_{n}$ in terms of maximal simple types of Bushnell and Kutzko [6]. We will use the standard notation from [6] and [18].

\subsection{Bessel functions}

We begin by recalling from [18, Section 5] the general formulation of Bessel functions. Let $\mathcal{K}$ be an open compact-modulo-center subgroup of $\mathrm{G}_{n}$ and let $\mathcal{U} \subset \mathcal{M} \subset \mathcal{K}$ be compact open subgroups of $\mathcal{K}$. Let $\tau$ be an irreducible smooth representation of $\mathcal{K}$ and let $\Psi$ be a linear character of $\mathcal{U}$. Take an open normal subgroup $\mathcal{N}$ of $\mathcal{K}$, which is contained in $\operatorname{Ker}(\tau) \cap \mathcal{U}$. Let $\chi_{\tau}$ be the (trace) character of $\tau$. The associated Bessel function $\mathcal{J}$ : $\mathcal{K} \rightarrow \mathbb{C}$ of $\tau$ is defined by

$$
\mathcal{J}(g):=[\mathcal{U}: \mathcal{N}]^{-1} \sum_{u \in \mathcal{U} / \mathcal{N}} \Psi\left(h^{-1}\right) \chi_{\tau}(g u)
$$

This is independent of the choice of $\mathcal{N}$. The basic properties of this Bessel function which we will need are given below.

Proposition 4.1 ([18, Proposition 5.3]). Assume that the data introduced above satisfy the following:

- $\left.\tau\right|_{\mathcal{M}}$ is an irreducible representation of $\mathcal{M}$; and

- $\left.\tau\right|_{\mathcal{M}} \cong \operatorname{Ind}_{\mathcal{U}}^{\mathcal{M}}(\Psi)$.

Then the Bessel function $\mathcal{J}$ of $\tau$ enjoys the following properties:

(i) $\mathcal{J}(1)=1$;

(ii) $\mathcal{J}(h g)=\mathcal{J}(g h)=\Psi(h) \mathcal{J}(g)$ for all $h \in \mathcal{U}$ and $g \in \mathcal{K}$;

(iii) if $\mathcal{J}(g) \neq 0$, then $g$ intertwines $\Psi$; in particular, if $m \in \mathcal{M}$, then $\mathcal{J}(m) \neq 0$ if and only if $m \in \mathcal{U}$;

When the representation $\tau$ is also unitarizable, the Bessel function enjoys another symmetry property, as in the finite field case in [17]. 
Lemma 4.2. In the situation of Proposition 4.1, assume further that $\tau$ is unitarizable.

Then

$$
\mathcal{J}(g)=\overline{\mathcal{J}\left(g^{-1}\right)}, \quad \text { for } g \in \mathcal{K}
$$

Proof. Note that $\Psi$ is unitary, since it is a character of the compact group $\mathcal{U}$. That is $\overline{\Psi\left(g^{-1}\right)}=$ $\Psi(g)$. Since $\chi_{\tau}$ is also unitary and $\chi_{\tau}(g h)=\chi_{\tau}(h g)$, for $g, h \in \mathcal{K}$, we get

$$
\begin{aligned}
\overline{\mathcal{J}\left(g^{-1}\right)} & :=[\mathcal{U}: \mathcal{N}]^{-1} \overline{\sum_{u \in \mathcal{U} / \mathcal{N}} \Psi\left(u^{-1}\right) \chi_{\tau}\left(g^{-1} u\right)} \\
& =[\mathcal{U}: \mathcal{N}]^{-1} \sum_{u \in \mathcal{U} / \mathcal{N}} \Psi(u) \chi_{\tau}\left(u^{-1} g\right) \\
& =[\mathcal{U}: \mathcal{N}]^{-1} \sum_{u \in \mathcal{U} / \mathcal{N}} \Psi(u) \chi_{\tau}\left(g u^{-1}\right) \\
& =[\mathcal{U}: \mathcal{N}]^{-1} \sum_{u \in \mathcal{U} / \mathcal{N}} \Psi\left(u^{-1}\right) \chi_{\tau}(g u) \\
& =\mathcal{J}(g), \text { for } g \in \mathcal{K} .
\end{aligned}
$$

The penultimate equality follows from the substitution $u \mapsto u^{-1}$ and the normality of $\mathcal{N}$ in $\mathcal{U}$.

\subsection{Maximal simple types}

Following [6, Section 6], the irreducible supercuspidal representations of $\mathrm{G}_{n}$ are classified by means of maximal simple types $(J, \lambda)$, where $J$ is a compact open subgroup of $\mathrm{G}_{n}$ and $\lambda$ is an irreducible representation of $J$. More precisely, $(J, \lambda)$ is introduced as follows. We refer to [6] for precise definitions of the objects introduced here.

Let $V=F^{n}$, an $n$-dimensional vector space over $F$ with standard basis. Thus we identify $\operatorname{Aut}_{F}(V)$ with $\mathrm{G}_{n}$ and $A=\operatorname{End}_{F}(V)$ with $\operatorname{Mat}_{n \times n}(F)$. Let $\mathfrak{A}$ be a principal hereditary $\mathfrak{o}_{F}$-order in $A$ with Jacobson radical $\mathfrak{P}$. Define $\mathbf{U}^{0}(\mathfrak{A})=\mathbf{U}(\mathfrak{A})=\mathfrak{A}^{\times}$and for $m \geqslant 1$, define $\mathbf{U}^{m}(\mathfrak{A})=1+\mathfrak{P}^{m}$. For $m \geqslant 0$, choose $\beta \in A$ such that $\beta \in$ $\mathfrak{P}^{-m} \backslash \mathfrak{P}^{1-m}, E=F[\beta]$ is a field extension of $F$, and $E^{\times}$normalizes $\mathfrak{A}$. Provided an additional technical condition is satisfied (namely $k_{F}(\beta)<0$ ), these data give a principal simple stratum $[\mathfrak{A}, m, 0, \beta]$ of $A$. Take $J=J(\beta, \mathfrak{A}), J^{1}=J^{1}(\beta, \mathfrak{A})$, and $H^{1}=H^{1}(\beta, \mathfrak{A})$ as defined in [6, Section 3]. Denote by $\mathcal{C}(\mathfrak{A}, \beta, \psi)$ the set of simple (linear) characters of $H^{1}$ as defined in [6, Section 3].

Recall from [6, Section 6] the following definition of maximal simple types.

Definition 4.3. The pair $(J, \lambda)$ is called a maximal simple type if one of the following holds:

(a) $J=J(\beta, \mathfrak{A})$ is an open compact subgroup associated to a simple stratum $[\mathfrak{A}, m, 0, \beta]$ of $A$ as above, such that, if $E=F[\beta]$ and $B=\operatorname{End}_{E}(V)$, then $\mathfrak{B}=\mathfrak{A} \cap B$ is a 
maximal $\mathfrak{o}_{E}$-order in $B$. Moreover, there exists a simple character $\theta \in \mathcal{C}(\mathfrak{A}, \beta, \psi)$ such that

$$
\lambda \cong \kappa \otimes \sigma
$$

where $\kappa$ is a $\beta$-extension of the unique irreducible representation $\eta$ of $J^{1}=J^{1}(\beta, \mathfrak{A})$, which contains $\theta$, and $\sigma$ is the inflation to $J$ of an irreducible cuspidal representation of

$$
J / J^{1} \cong \mathbf{U}(\mathfrak{B}) / \mathbf{U}^{1}(\mathfrak{B}) \cong \mathrm{GL}_{r}\left(\mathfrak{k}_{E}\right)
$$

where $r=n /[E: F]$.

(b) $(J, \lambda)=(\mathbf{U}(\mathfrak{A}), \sigma)$, where $\mathfrak{A}$ is a maximal hereditary $\mathfrak{o}_{F}$-order in $A$ and $\sigma$ is the inflation to $\mathbf{U}(\mathfrak{A})$ of an irreducible cuspidal representation of

$$
\mathbf{U}(\mathfrak{A}) / \mathbf{U}^{1}(\mathfrak{A}) \cong \mathrm{GL}_{n}\left(\mathfrak{k}_{F}\right)
$$

We will regard case (b) formally as a special case of case (a) by setting $\beta=0$ and $E=$ $F$, and $\theta, \eta, \kappa$ trivial. In either case, we put $\mathbf{J}=E^{\times} J$. With these data, any irreducible supercuspidal representation $\pi$ of $\mathrm{G}_{n}$ is of the form

$$
\pi \cong \mathrm{c}-\operatorname{Ind}_{\mathbf{J}}^{\mathrm{G}_{n}}(\Lambda)
$$

for some choice of $(\mathbf{J}, \Lambda)$, where $\left.\Lambda\right|_{J}=\lambda$. We call such a pair $(\mathbf{J}, \Lambda)$ and extended maximal simple type.

For $\pi$ an irreducible supercuspidal representation of $\mathrm{G}_{n}$, any two extended maximal simple types in $\pi$ are conjugate in $\mathrm{G}_{n}$. This fact allows one to associate some invariants to $\pi$. The simple character $\theta$ in the construction of an extended maximal simple type for $\pi$ determines an endo-class $\Theta=\Theta(\pi)$ as defined in [2]. We do not recall precisely the definition of endo-class: it is a class for a certain equivalence relation on functions which take values in simple characters. For $i=1$, 2, let $\theta_{i} \in \mathcal{C}\left(\mathfrak{A}_{i}, \beta_{i}, \psi\right)$ be simple characters for $G_{n}$. If $\theta_{1}, \theta_{2}$ have the same endo-class then they intertwine in $G_{n}$; if, moreover, the hereditary orders $\mathfrak{A}_{1}, \mathfrak{A}_{2}$ are isomorphic then $\theta_{1}, \theta_{2}$ are conjugate in $\mathrm{G}_{n}$.

Although the field extension $E / F$ involved in the construction of a maximal simple type in $\pi$ is not uniquely determined, its residue degree and ramification index are in fact invariants of the endo-class $\Theta=\Theta(\pi)$ and we write

$$
f(\Theta)=f(E / F), e(\Theta)=e(E / F), \operatorname{deg}(\Theta)=[E: F]
$$

These are then also invariants of $\pi$ so we write $\operatorname{deg}(\pi)=\operatorname{deg}(\Theta)$ and call it the degree of $\pi$. We also remark that the $\mathfrak{o}_{F}$-period of the hereditary order $\mathfrak{A}$ in the construction of any maximal simple type in $\pi$ is $e(\Theta)$. 


\subsection{Explicit Whittaker functions}

Let $\pi$ be an irreducible unitarizable supercuspidal representation of $\mathrm{G}_{n}$. By [3, Proposition 1.6], there is an extended maximal simple type $(\mathbf{J}, \Lambda)$ in $\pi$ such that

$$
\operatorname{Hom}_{U_{n} \cap \mathbf{J}}\left(\psi_{n}, \Lambda\right) \neq 0 \text {. }
$$

Since $\Lambda$ restricts to a multiple of some simple character $\theta \in \mathcal{C}(\mathfrak{A}, \beta, \psi)$, one obtains that $\theta(u)=\psi_{n}(u)$ for all $u \in \mathrm{U}_{n} \cap H^{1}$. As in [18, Definition 4.2], one defines a character $\Psi_{n}:\left(J \cap \mathrm{U}_{n}\right) H^{1} \rightarrow \mathbb{C}^{\times}$by

$$
\Psi_{n}(u h):=\psi_{n}(u) \theta(h)
$$

for all $u \in J \cap \mathrm{U}_{n}$ and $h \in H^{1}$. By [18, Theorem 4.4], the data

$$
\mathcal{K}=\mathbf{J}, \tau=\Lambda, \mathcal{M}=\left(J \cap \mathrm{P}_{n}\right) J^{1}, \mathcal{U}=\left(J \cap \mathrm{U}_{n}\right) H^{1}, \text { and } \Psi=\Psi_{n}
$$

satisfy the conditions in Proposition 4.1 and hence define a Bessel function $\mathcal{J}$.

Now we define a function $W_{\pi}: \mathrm{G}_{n} \rightarrow \mathbb{C}$ by

$$
W_{\pi}(g):= \begin{cases}\psi_{n}(u) \mathcal{J}(j) & \text { if } g=u j \text { with } u \in \mathrm{U}_{n}, j \in \mathbf{J}, \\ 0 & \text { otherwise, }\end{cases}
$$

which is well-defined by Proposition 4.1(ii). Then, by [18, Theorem 5.8], $W_{\pi}$ is a Whittaker function for $\pi$. Moreover, since $\pi$ is unitarizable, the same is true of $\Lambda$, so $W_{\pi}$ is a $\mathbf{J}$-special Whittaker function for $\pi$, by Lemma 4.2. By Proposition 4.1, the restriction of $W_{\pi}$ to $\mathrm{P}_{n}$ has a particularly simple description: for $g \in \mathrm{P}_{n}$,

$$
W_{\pi}(g)= \begin{cases}\Psi_{n}(g) & \text { if } g \in\left(J \cap \mathrm{U}_{n}\right) H^{1} \\ 0 & \text { otherwise }\end{cases}
$$

\subsection{Proof of Proposition 1.6}

Let $\pi_{1}, \pi_{2}$ be irreducible unitarizable supercuspidal representations of $\mathrm{G}_{n}$ with the same endo-class $\Theta$. We will use all the notation of Definition 4.3 but with subscripts ${ }_{1}, 2$.

Let $\left(\mathbf{J}_{1}, \Lambda_{1}\right)$ be an extended maximal simple type in $\pi_{1}$ such that

$$
\operatorname{Hom}_{\mathrm{U}_{n} \cap \mathbf{J}_{1}}\left(\psi_{n}, \Lambda_{1}\right) \neq 0 \text {. }
$$

By [18, Remark 4.15], we may assume that the pair $\left(\mathrm{U}_{n}, \psi_{n}\right)$ arises from the construction of [18, Theorem 3.3]. This construction, which produces a particular maximal unipotent subgroup and non-degenerate character, depends only on the simple character $\theta_{1}$. Thus, by [18, Corollary 4.13], the space $\operatorname{Hom}_{\mathrm{U}_{n} \cap \mathbf{J}_{1}}\left(\psi_{n}, \Lambda\right)$ is non-zero for any extended maximal simple type $\left(\mathbf{J}_{1}, \Lambda\right)$ containing $\theta_{1}$. 
Now let $\left(\mathbf{J}_{2}, \Lambda_{2}\right)$ be any extended maximal simple type in $\pi_{2}$. The hereditary orders $\mathfrak{A}_{i}$ have the same period $e(\Theta)$ so are conjugate in $\mathrm{G}_{n}$; replacing $\mathfrak{A}_{2}$ by a conjugate if necessary, we assume they are equal. Then the simple characters $\theta_{1}, \theta_{2}$ are conjugate in $G_{n}$, by definition of endo-equivalence; again, replacing $\theta_{2}$ by a conjugate if necessary, we assume they are equal. Now $\mathbf{J}_{i}$ is the $G_{n}$-normalizer of $\theta_{i}$ so we have $\mathbf{J}_{1}=\mathbf{J}_{2}$. Hence, by the remarks above,

$$
\operatorname{Hom}_{\mathrm{U}_{n} \cap \mathbf{J}_{1}}\left(\psi_{n}, \Lambda_{2}\right) \neq 0 \text {. }
$$

Thus the characters $\Psi_{n}^{1}, \Psi_{n}^{2}$ as defined in (4.4) are equal. Finally, by (4.6), the $\mathbf{J}_{1}$-special Whittaker functions $W_{\pi_{1}}, W_{\pi_{2}}$ defined by (4.5) agree on $\mathrm{P}_{n}$. Thus $\left(W_{\pi_{1}}, W_{\pi_{2}}\right)$ is a special pair for $\left(\pi_{1}, \pi_{2}\right)$, which completes the proof of Proposition 1.6.

Remark 4.7. In the proof of the existence of a special pair, we do not in fact use that the endo-classes for $\pi_{1}, \pi_{2}$ coincide, but only that $\mathbf{J}_{1}, \mathbf{J}_{2}$ are contained in a common compact-modulo-center open subgroup of $\mathrm{G}$, that $H_{1}^{1} \cap \mathrm{P}_{n}=H_{2}^{1} \cap \mathrm{P}_{n}$ and that $\theta_{1}, \theta_{2}$ coincide on $H_{1}^{1} \cap \mathrm{P}_{n}$. This is significantly weaker: for example, if $\operatorname{deg}\left(\pi_{1}\right)=n$ and $\beta_{1}$ is a minimal element (see, for example, [6, Section 1.4] for the definition) then $H_{1}^{1} \cap \mathrm{P}_{n}=$ $U^{\left[\frac{m}{2}\right]+1}\left(\mathfrak{A}_{1}\right) \cap \mathrm{P}_{n}$.

\section{Conductors of pairs}

In this section we will prove Corollary 1.7 . The techniques here are entirely different, relying on the explicit computation of conductors of pairs of supercuspidal representations from [5] and their application in [4].

\subsection{Endo-classes}

In [4], Bushnell and Henniart define a function $\mathfrak{F}$ of pairs of endo-classes with the property that, for $\pi, \tau$ irreducible supercuspidal representations of $\mathrm{G}_{n}, \mathrm{G}_{r}$ respectively, with $n>$ $r \geqslant 1$, the conductor satisfies

$$
f(\pi \times \widetilde{\tau}, \psi)=n r(\mathfrak{F}(\Theta(\pi), \Theta(\tau))+1) .
$$

Moreover, [4, Theorem C] says that this function characterizes endo-classes in the following way: for endo-classes $\Theta_{1}, \Theta_{2}$,

$$
\mathfrak{F}\left(\Theta_{1}, \Theta_{2}\right) \geqslant \mathfrak{F}\left(\Theta_{1}, \Theta_{1}\right),
$$

with equality if and only if $\Theta_{1}=\Theta_{2}$.

The final ingredient we need is that, given an endo-class $\Theta$, there is an irreducible supercuspidal representation $\tau$ of $\mathrm{G}_{r}$ with endo-class $\Theta$ whenever $r$ is a multiple of the degree $\operatorname{deg}(\Theta)$. This is immediate from the definitions of endo-class in [3] and of maximal simple types. 


\subsection{Proof of Corollary 1.7}

Let $\pi_{1}, \pi_{2}$ be unitarizable irreducible supercuspidal representations of $\mathrm{G}_{n}$, with endoclasses $\Theta_{1}, \Theta_{2}$ respectively, and suppose that $\operatorname{deg}\left(\pi_{1}\right)<n$. Suppose the local gamma factors $\gamma\left(s, \pi_{1} \times \tau, \psi\right)$ and $\gamma\left(s, \pi_{2} \times \tau, \psi\right)$ are equal as functions in the complex variable $s$, for all irreducible supercuspidal representations $\tau$ of $\mathrm{G}_{r}$ with $r=1, \ldots,\left[\frac{n}{2}\right]$.

Put $r:=\operatorname{deg}\left(\pi_{1}\right)$, which is a proper divisor of $n$; in particular, $r \leqslant\left[\frac{n}{2}\right]$. Let $\tau$ be an irreducible supercuspidal representation $\tau$ of $G_{r}$ with endo-class $\Theta(\tau)=\Theta_{1}$. Then, by (5.1) and hypothesis, we have

$$
\mathfrak{F}\left(\Theta_{1}, \Theta_{1}\right)=\frac{f\left(\pi_{1} \times \tilde{\tau}, \psi\right)}{n r}-1=\frac{f\left(\pi_{2} \times \widetilde{\tau}, \psi\right)}{n r}-1=\mathfrak{F}\left(\Theta_{2}, \Theta_{1}\right) .
$$

We deduce, from (5.2), that $\Theta_{1}=\Theta_{2}$. Then Proposition 1.6 and Theorem 1.5 combine to imply that $\pi_{1}$ is equivalent to $\pi_{2}$.

Remark 5.3. The restriction of a simple character $\theta \in \mathcal{C}(\mathfrak{A}, \beta, \psi)$ to the groups $H^{t+1}=$ $H^{1} \cap \mathbf{U}^{t+1}(\mathfrak{A}), t \geqslant 0$, determines a family of endo-classes. By considering these endoclasses, rather than just those coming from the simple character $\theta$, it seems likely that one could prove a more general version of Corollary 1.7 by generalizing the function $\mathfrak{F}$.

However, even in the most optimistic scenario, this will leave the case where, for $i=$ 1,2 , we have $\operatorname{deg}\left(\pi_{i}\right)=n$ and any simple character $\theta_{i} \in \mathcal{C}\left(\mathfrak{A}, \beta_{i}, \psi\right)$ in $\pi_{i}$ has $\beta_{i}$ a minimal element. One would need to prove that the equality of local gamma factors implies that one can assume $\beta_{1} \equiv \beta_{2}\left(\bmod \mathfrak{P}^{-\left[\frac{m}{2}\right]}\right)$ to enable us to construct a special pair of Whittaker functions (see Remark 4.7). However, even the case $n=3$ seems to be very difficult to analyze directly via the explicit construction of supercuspidal representations, even in the tame case.

Acknowledgments. The work of the first-named author is supported in part by NSF grants DMS-1001672 and DMS-1301567. Both the first and the second-named authors would like to thank the Chinese Academy of Sciences for invitation and support over the years when their research work related to this paper was in process. The second-named author is supported by NSC101-2918-I-006-003 and would like to thank the University of East Anglia for providing a stimulating environment during the execution period of the project. The third-named author is supported by EPSRC grant EP/H00534X/1. We thank the referee for some helpful comments, in particular for encouraging us to remove an unnecessary hypothesis on the characteristic.

\section{References}

[1] Bernšteŭn, I. N., Zelevinskiŭ, A. V.: Representations of the group $G L(n, F)$, where $F$ is a local non-Archimedean field, Uspehi Mat. Nauk 31, no. 3 (189), 5-70 (1976) (in Russian); English transl.: Russian Math. Surveys 31, no. 3, 1-68 (1976)

[2] Bushnell, C. J., Henniart, G.: Local tame lifting for GL $(N)$. I. Simple characters, Inst. Hautes Études Sci. Publ. Math. no. 83, 105-233 (1996) 
[3] Bushnell, C. J., Henniart, G.: Supercuspidal representations of GL : $_{n}$ explicit Whittaker functions, J. Algebra 209, 270-287 (1998)

[4] Bushnell, C. J., Henniart, G.: Local tame lifting for GL $(n)$. IV. Simple characters and base change, Proc. London Math. Soc. (3) 87, 337-362 (2003)

[5] Bushnell, C. J., Henniart, G. M., Kutzko, P. C.: Local Rankin-Selberg convolutions for $\mathrm{GL}_{n}$ : explicit conductor formula, J. Amer. Math. Soc. 11, 703-730 (1998)

[6] Bushnell, C. J., Kutzko, P. C.: The admissible dual of GL $(N)$ via compact open subgroups, Annals of Mathematics Studies, vol. 129, Princeton University Press, Princeton, NJ (1993)

[7] Chen, J.-P. J.: Local Factors, Central Characters, and Representations of the General Linear Group over Non-Archimedean Local Fields, Thesis, Yale University (1996)

[8] Chen, J.-P. J.: The $n \times(n-2)$ local converse theorem for $\operatorname{GL}(n)$ over a $p$-adic field, J. Number Theory 120, 193-205 (2006)

[9] Cogdell, J. W., Piatetski-Shapiro, I. I.: Converse theorems for GL ${ }_{n}$. II, J. Reine Angew. Math. 507, 165-188 (1999)

[10] Gel'fand, I. M., Kazhdan, D. A.: Representations of the group GL $(n, K)$ where $K$ is a local field, In: Lie groups and their representations (Proc. Summer School, Bolyai János Math. Soc., Budapest, 1971), Halsted, New York, 95-118 (1975)

[11] Henniart, G.: Caractérisation de la correspondance de Langlands locale par les facteurs $\epsilon$ de paires, Invent. Math. 113, 339-350 (1993)

[12] Jacquet, H., Langlands, R. P.: Automorphic forms on GL(2), Lecture Notes in Mathematics, Vol. 114, Springer-Verlag, Berlin (1970)

[13] Jacquet, H., Piatetski-Shapiro, I. I., Shalika, J. A.: Rankin-Selberg convolutions, Amer. J. Math. 105, 367-464 (1983)

[14] Jacquet, H., Piatetski-Shapiro, I. I., Shalika, J. A.: Automorphic forms on GL(3). I, Ann. of Math. (2) 109, 169-212 (1979)

[15] Jacquet, H., Shalika, J.: A lemma on highly ramified $\epsilon$-factors, Math. Ann. 271, 319-332 (1985)

[16] Jiang, D., Soudry, D.: The local converse theorem for $\mathrm{SO}(2 n+1)$ and applications, Ann. of Math. (2) 157, 743-806 (2003)

[17] Nien, C.: A proof of finite field analogue of Jacquet's conjecture, Amer. J. Math. 136, 653-674 (2014)

[18] Paškūnas, V., Stevens, S.: On the realization of maximal simple types and epsilon factors of pairs, Amer. J. Math. 130, 1211-1261 (2008) 
[19] Shahidi, F.: Fourier transforms of intertwining operators and Plancherel measures for GL $(n)$, Amer. J. Math. 106, 67-111 (1984)

[20] Zelevinsky, A. V.: Induced representations of reductive $\mathfrak{p}$-adic groups. II. On irreducible representations of GL(n), Ann. Sci. École Norm. Sup. (4) 13, 165-210 (1980) 